\title{
Ectopic expression of rice OsMADS1 reveals a role in specifying the lemma and palea, grass floral organs analogous to sepals
}

Received: 28 August 2000 / Accepted: 14 February 2001 / Published online: 25 April 2001

(C) Springer-Verlag 2001

\begin{abstract}
MADS-domain-containing transcription factors play diverse roles in plant development. The prototypic members of this gene family are the floral organ identity genes of the model dicotyledonous plant, Arabidopsis thaliana. Sequence relatedness and function ascribe them to AP1/AGL9, AG, AP3 and PI gene groups. The rice MADS-box gene, OsMADS1, is a member of the AP1/ AGL9 sub-group. Tomato and Petunia members of this sub-group specify floral meristem identity and control organ development in three inner whorls. Reported here are phylogenetic analyses that show OsMADS1 to form a distinct clade within the AGL9 gene family. This sub-group currently has only three other monocot genes. We have studied the expression pattern of OSMADS1 and determined the consequences of its ectopic expression in transgenic rice plants. OsMADS1 is not expressed during panicle branching; earliest expression is in spikelet meristems where it is excluded from the outer rudimentary/sterile glumes. During organogenesis, OSMADS1 expression is confined to the lemma and palea, with weak expression in the carpel. Ectopic OSMADS1 expression results in stunted panicles with irregularly positioned branches and spikelets. Additionally, in spikelets, the outer rudimentary glumes are transformed to lemma/palea-like organs. Together, these data suggest a distinct role for OsMADS1 and its monocot relatives in assigning lemma/palea identity.
\end{abstract}

Edited by G. Jürgens

K. Prasad · P. Sriram · K. Kushalappa · U. Vijayraghavan $(\bowtie)$

Department of Microbiology and Cell Biology,

Indian Institute of Science, Bangalore 560012, India

e-mail: uvr@mcbl.iisc.ernet.in

Tel.: +91-80-3092681, Fax: +91-80-3602697

C.S. Kumar

The Institute for Molecular Agrobiology,

The National University of Singapore, 117604, Singapore

Present address:

K. Kushalappa, Department of Botany,

University of British Columbia, Vancouver, Canada
Keywords OsMADS1 - MADS-box genes · Flower development $\cdot$ Lemma/palea $\cdot$ Phylogeny

\section{Introduction}

The shoot apical meristem of higher plants contains undifferentiated progenitor cells that give rise to both vegetative and reproductive structures. Transition from vegetative to reproductive phase is controlled by both genetic and environmental factors and is responsible for specifying floral identity to newly arising meristems. The flowers formed hence are complex reproductive structures wherein the invariant ordering of floral organs - sepals, petals, stamens and carpels from the periphery to the center - implies a common ground plan for organ patterning in all flowering plants. In the model dicot plant Arabidopsis thaliana, conferment of floral fate requires the activity of meristem identity genes: $L F Y, A P 1, A P 2$ and CAL (Weigel et al. 1992; Bowman et al. 1993; Weigel 1995). In addition, these floral meristem identity genes are transcription activators of floral organ identity genes that pattern organs in four concentric whorls (Parcy et al. 1998; Wagner et al. 1999). The latter genes include $A P 1, A P 2, A P 3 / P I$ and $A G$, identified first on the basis of loss-of-function homeotic mutations that alter cell fate in two adjacent whorls of the flower (reviewed by Ma 1994; Irish 1999). Thus, their combined and individual domains of action are proposed to dictate organ fate. All but one of these A. thaliana floral organ identity genes belong to the MADS-domain-containing group of evolutionarily conserved transcription factors that regulate gene expression in plant, yeast and animal cells (Reichmann and Meyerowitz 1997). The temporal and spatial expression pattern of the floral organ identity genes is consistent with their function in two adjacent whorls (Yanofsky et al. 1990; Jack et al. 1992; Mandel et al. 1992b; Goto and Meyerowtiz 1994). For instance, $A G$ is expressed in whorls 3 and 4 of the flower and loss-of$A G$ function results in altered cell fate in these whorls alone (Yanofsky et al. 1990). The sufficiency of these 
genes to alter cell fate, when expressed ectopically in floral whorls where they are not normally found, has also been tested (Mandel et al. 1992a; Mizukami and Ma 1992; Jack et al.1994). All of these data are, to a large extent, explained and accommodated by the ABC model for floral organ specification, originally proposed based on the phenotypes of loss-of-function mutations in these floral organ identity genes (Coen and Meyerowitz 1991; Ma 1994; Irish 1999).

The MADS-box genes have provided a gateway to probing the function of regulatory transcription factors in patterning floral organs in diverse species. They also provide tools to study mechanisms by which sequence homologues and/or orthologues could contribute to species-specific differences in floral organ patterns. Modern day monocotyledonous (monocot) plants shared ancestors with dicotyledonous (dicots) plants more than 120 million years ago (Doyle 1973). The divergent and reduced flowers of cereal grasses, a large group among the monocots, provide an excellent model to examine the above mentioned hypotheses. Typical of grasses, rice flowers are borne on a branched inflorescence called the panicle, where the developmental progress from inflorescence specification to flower meristem allocation occurs in steps. Upon floral induction, the inflorescence meristem generates primary branch primordia on its flanks, which in turn elongate and allocate basally second order branch primordia. Subsequently, spikelet primordia are specified apically on the primary as well as on the secondary branch primordia (Hoshikawa 1989). In sum, the inflorescence/panicle meristem, primary branch primordia, secondary branch primordia, and floral primordia comprise the reproductive meristems in rice. A rice spikelet primordium bears a single flower in the axil of rudimentary glumes and the flower contains the organslemma, palea, lodicules, stamens and carpel. Recently, several rice MADS-box genes have been partially characterized (Chung et al. 1994, 1995; Kang et al. 1995, 1998; Kang and An 1997). However, their precise role during panicle development remains largely unknown. One such MADS-box gene is OsMADS1, whose predicted product has overall $68.4 \%$ identity to AGL9, 56.2\% identity to AGL2, and $44.4 \%$ identity to AP1 proteins of Arabidopsis (Chung et al. 1994). All of these Arabidopsis genes are initially expressed throughout the floral meristem. Their later expression patterns vary; they are either expressed in all four floral organ primordia, or only in the perianth, or in three inner whorls. Preliminary studies on OSMADS1 reveal it to be temporally and spatially regulated, with features both similar to and different from AP1/AGL9-like factors. Early expression is uniform throughout the floral meristem and its later high level expression is restricted to the lemma and palea (modified first whorl organs), with weak expression in the carpel (Chung et al. 1994; Vijayraghavan 1996). We have examined the phylogenetic relationships of $O S M$ $A D S 1$ and its closely related homologue OsMADS5. We find that they form a distinct clade within the AP1/AGL9 group with no members as yet from any dicot species that can be assigned to this sub-group. Also, we have studied in detail the expression pattern of OsMADS1 and have determined that it is not expressed in the spikelet organs, i.e. the glumes, which are modified bracts peripheral to the flower. Additionally, by adopting a reverse genetics strategy, we have studied the consequences of ectopic OSMADS1 expression to understand its role in organ fate specification. We show that OsMADS1 has a role in assigning cells to a lemma and palea fate, and that its ectopic expression affects panicle branch differentiation.

\section{Materials and methods}

Phylogenetic analysis

The nucleotide and protein sequence of OSMADS1 was retrieved from the GenBank sequence database (Accession Number L34271). The predicted protein from the full-length cDNA sequence was used as query in BLAST and FASTA searches for related sequences in the GenBank and SwissProt non-redundant sequence databases. The high scoring sequences were retrieved from the databases. Plant MADS-box genes used for phylogenetic analysis are given below, with their accession numbers given within brackets. Rice genes, OsMADS1 (L34271), OsMADS2 (L37526), OsMADS3 (L37528), OsMADS4 (L37527), OsMADS5 (U78890), OsMADS6 (U78782), OsMADS14 (AF058697), OsMADS15 (AF058698), OsMADS16 (AF077760), OsMADS17 (AF109153), OsMADS45 (U31994), RAP1b (AB041020), rice MADS-like (RML; (AB003324); Arabidopsis genes AGL2 (B39534), AGL3 (P29383), AGL4 (P29384), AGL6 (M55554), AGL9 (AF015552), AGL13 (U20183), AP1 (Z16421), AGAMOUS (S10933); and maize genes ZAP1 (L46400), ZAG1 (L18924), ZAG2 (X80206), ZMM1 (X81199), ZMM2 (L81162), ZMM3 (Y09301), ZMM8 (Y09308) and SILKY1 (AF181479) were retrieved from GenBank. The sequences for tomato TDR5 (X60480), petunia FBP2 (M91666) and Antirrhinum PLENA (S53900) genes were also retrieved from GenBank. Yeast MCMI gene (P11746) and human $M E F 2$ (L16794) gene sequences were also similarly retrieved.

\section{Alignment and sequence analysis}

For alignment and sequence analysis the following approaches were taken. The full-length protein sequences were aligned using the PILEUP of the UWGCG package. This was refined using CLUSTALW 1.7. Phylogenetic analysis, with the full-length protein sequence, employing maximum parsimony methods performed using the experimental version of PAUP* program, (4.0.0d55 Version for UNIX; D.L. Swofford, Laboratory of Molecular Systematics, Smithsonian Institute, Washington, D.C). For maximum parsimony analysis 33 taxa were analyzed, with 100 replicates, keeping all optimal trees in each replicate. Gaps were treated as missing residues. Tree construction was undertaken using the Bootstrap method with Neighbor-Joining (NJ) or Heuristic search. The NJ analysis was done using the software default settings and the optimality criterion was set to distance. The trees were examined using the PAUPDISPLAY program of the UWGCG package. The tree obtained using Bootstrap method with heuristic search algorithm was essentially the same as that obtained with NJ. The MCM1 protein sequence was used as outgroup in this analysis, to study the branching order of the gene lineages relative to the main group. In addition, a tree was also generated with the plant floral organ identity gene $A G$ as the outgroup. Distance based criteria (Kimura protein distance) and NJ were used to generate the tree.

Furthermore, in the aligned predicted protein sequences, the 525 nucleotides coding for the MADS-intermediate-keratin-like 
(MIK) region of OsMADS1 and related proteins were visually selected, manually refined and analyzed. These nucleotide sequences were re-aligned using the PILEUP program. The 120 nucleotides corresponding to the MADS box and 233 nucleotides corresponding to the $\mathrm{K}$ box sequences were thus deciphered. Sequence divergence at the nucleotide level was then derived using the DISTANCES program of the UWGCG package. The Tajima and Nei method (Tajima and Nei 1984) was used to estimate rate of nucleotide substitution. The resulting distance matrix was processed using the GROWTREE program of the UWGCG package. The tree was constructed using the NJ method. The trees were visualized with TREEVIEW. The DIVERGE program of the UWGCG package based on the methods described by Li et al. (1985) and $\mathrm{Li}$ (1993) was employed to determine the rates of nonsynonymous substitution in the MADS box, $\mathrm{K}$ box and MIK region.

Isolation of OsMADS1 cDNA clone

A $\lambda$ cDNA library in the vector $\lambda$ UniZAP (Stratagene), representing mRNAs from wild-type rice panicles (length 2-5 cm; Kushalappa 1999), was screened to obtain full-length OsMADS1 cDNAs. The probe used was a partial cDNA, previously cloned in the laboratory that encoded amino acid residues $175-257$ of the predicted OsMADS1 protein (Chung et al. 1994; Vijayraghavan 1996). From screening $10^{6}$ plaques, 12 positives were obtained, one of which contained the full-length cDNA as determined by sequence analysis of the excised phagemid.

Construction of transgene to ectopically express OsMADS1

Binary vector pCAMBIA1300 was modified for ectopic expression of $O S M A D S 1$. The complete maize ubiquitin (Ubi1) promoter (Christensen et al. 1992) was cloned in as a HindIII-BamHI fragment. Subsequently, the nopaline synthase (nos) terminator was cloned as a $S a c \mathrm{I}-E c o$ RI fragment downstream of the Ubi1 promoter. Lastly, OsMADS1 full-length cDNA was blunt-end cloned into the $S a c$ I site between the promoter and terminator sequences. The sense orientation of the cDNA was verified by KpnI digestion. The recombinant was called pUbi-OsMADS1.

Rice transformation

The embryogenic calli from rice seeds of the japonica variety TP309 were co-cultivated with Agrobacterium tumefaciens (Hiei et al. 1994) carrying the pUbi-OSMADS1 plasmid. Co-cultivation was for 2-3 days in the dark (at $25^{\circ} \mathrm{C}$ ) on $2 \mathrm{~N} 6$-AS medium supplemented with $100 \mu \mathrm{M}$ acetosyringone. The co-cultivated calli were washed with water containing $250 \mathrm{mg} / \mathrm{l}$ cefotaxime and then transferred onto N6 medium containing $50 \mathrm{mg} / \mathrm{l}$ hygromycin and $250 \mathrm{mg} / \mathrm{l}$ cefotaxime for 8 weeks. Actively proliferating calli were further transferred onto regeneration medium $(3 \mathrm{mg} / \mathrm{l} \mathrm{BAP}$ and $0.5 \mathrm{mg} / \mathrm{l} \mathrm{NAA}$ ) for 4-8 weeks and subsequently onto $1 / 2 \mathrm{MS}$ medium (0.05 mg/l NAA) for 3 weeks. A light/dark cycle of $16 / 8 \mathrm{~h}$ was provided during regeneration and rooting. Plantlets were then transferred to soil and grown in a greenhouse.

\section{Light microscopy and scanning electron microscopy}

Morphological features of transgenic and wild-type panicles and flowers were observed by stereomicroscopy. For SEM, panicles were dissected away from the enveloping leaf sheaths, fixed, dehydrated and then critical point dried. The dried panicles were mounted onto stubs, sputter coated with gold and then viewed in a Joel JSM-5310 LV microscope (Skandinaviska AB, Sundbyerg, Sweden).

\section{RT-PCR of OsMADS1 transcripts}

Total RNA from young leaves of transgenic plants or control plants was isolated by phenol extraction and then treated with RNAse-free DNAse. RT-PCR experiments were performed using this total RNA (Wang and Tobin 1998; Nandi et al. 2000). First strand cDNA was made from $7.5 \mu \mathrm{g}$ RNA in $20 \mu \mathrm{l}$ reaction mixture using the primer OSM2-1 (5' CGGGATCCGCCAATTAATTGTTACC 3'). The primer used in control cDNA synthesis reactions was Act2 (5' GATGGATCCTCCAATCCAGACACTGTA $\left.3^{\prime}\right)$. Standard PCR reactions of $50 \mu \mathrm{l}$ were then performed with $5 \mu \mathrm{RT}$ product generated for OsMADS1 or ACT1. PCRs contained, as the forward primer, OSM4 (5' GAGAATGTGCTCCATATG $\left.3^{\prime}\right)$ in the OsMADS1 RT-PCR or Act1 (5' GTATTGTGTTGGACTCTGGTGATGGTGT $3^{\prime}$ ) in the ACT1 RT-PCR reactions. PCR products were visualized on $1 \%$ agarose gel after loading $10 \mu \mathrm{l}$ from each PCR reaction.

In situ hybridization for OsMADS1 transcripts

Preparation of rice tissues and hybridization conditions included only minor modifications of the protocol as described by Drews et al. (1991) for Arabidopsis. The OsMADS1 ${ }^{35} \mathrm{~S}$-labeled anti-sense mRNA probe was synthesized with T3 RNA polymerase from a BamHI digest of a plasmid containing $500 \mathrm{bp}$ of the $3^{\prime}$ gene specific region of OSMADS1. The slides were exposed for 1 week.

\section{Results and discussion}

\section{Phylogenetic relationships of OsMADS1}

A preliminary sequence alignment of the predicted OsMADS1 protein to a few other plant MADS-domain-containing factors had suggested relatedness to the AGL2 and APl genes of Arabidopsis (Chung et al. 1994). This homology was consistent with its flower-specific expression in the lemma, palea and carpel (Chung et al. 1994; Vijayraghavan 1996). Members of the plant MADSdomain gene family can be distinguished by phylogenetic relationships, and such analyses show them to cluster into distinct groups: $A P 3 / P I, A G, A P 1 / A G L 9$ and orphan gene clades (Purugganan et al. 1995; Theissen et al. 1996; Alvarez-Buylla et al. 2000). Unlike members of the $A P 3$ or $P I$ groups, the precise function of many of the molecules belonging to the API/AGL9 clades remains unknown. Additionally, this group bears within it multiple lineages, the most obvious being AP1, AGL9 and the AGL6/AGL13 clades.

Recently, several MADS-domain-containing genes have been isolated, their expression patterns studied and in some instances in vivo functions deciphered through reverse genetic approaches (Reichmann and Meyerowitz 1997; Schmidt and Ambrose 1998). We have attempted to obtain a hint of OsMADS1 function by examining its evolutionary relationship with other genes, particularly those of the AP1/AGL9 gene clades. The related evolutionary histories of individual gene groups could reflect their distinct functional specificity in regulating floral cell fate. The amino acids corresponding to the full length predicted OsMADS1 protein align well with predicted proteins for most members of this group particularly in the following domains: MADS box, intermediate linker 
Fig. 1 A Phylogenetic tree for the rice OsMADS1, OsMADS5 and $A P 1, A G L 9$-like MADSbox genes from other genera. A selected number of $A G$-related genes were also used in the analysis. Maximum parsimony based analyses was performed for the predicted full-length protein sequences. The yeast MCM1 protein was used as the outgroup. Numbers (written above each node) give the Bootstrap values from 100 replicates. Nodes with $<50 \%$ bootstrap support are collapsed. The tree length is 2,408 steps. The topology of the tree remained the same even when the Heuristic search algorithm was employed. The shaded box indicates the OsMADS1 clade.

B Neighbor-Joining-based tree showing evolutionary distances for plant MADS-box-containing genes. The scale bar indicates the number of nucleotide substitutions. The distance matrix was determined for nucleotide sequences corresponding to the MADS-intermediate-keratin-like (MIK) domains of the indicated proteins, by determining the Tajima-Nei nucleotide substitution rate

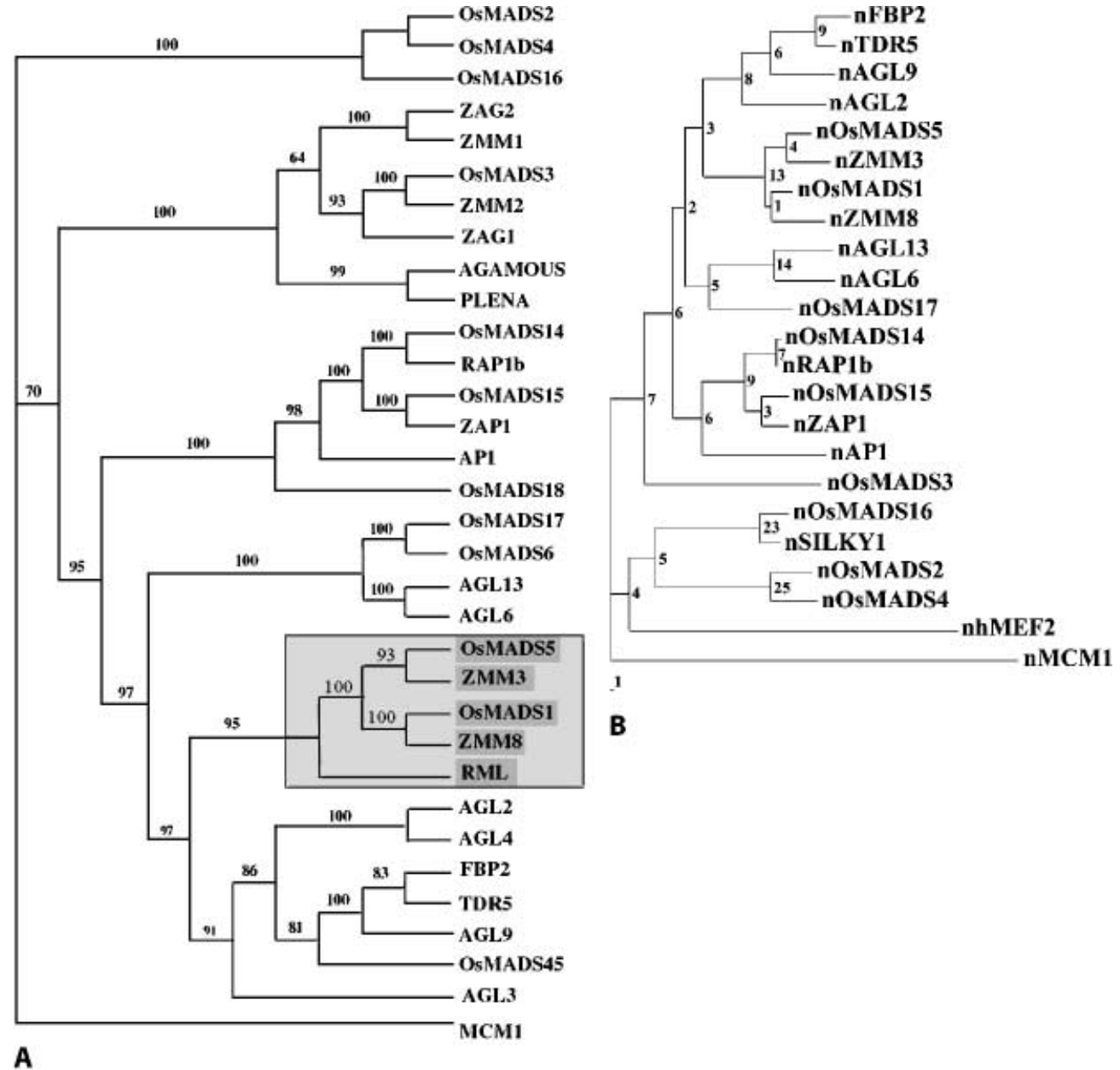

region and the K box. These regions comprise the DNA binding domain, connecting linker or intermediate domain and the predicted coiled-coil domain with structural similarity to the fibrous protein keratin, respectively. The alignment was unambiguous for protein as well as the nucleotide sequences that code for the MIK region of the proteins (data not shown). The phylogenetic relatedness of OSMADS1 to members of the API and AGL9 clades from Arabidopsis, Petunia, tomato, rice and maize was examined. In addition, relationship to members of the $A G$ clade from Arabidopsis, Antirrhinum, rice and maize was also determined. We employed the maximum parsimony method (MP) and phylogenetic trees were generated by $\mathrm{NJ}$ to determine its position within this gene family. A single tree was derived from parsimony analysis with high bootstrap support in 100 replicates [consistency in$\operatorname{dex} \mathrm{CI}=0.6952$, retention index $=0.6732$, rescaled consistency index $(\mathrm{RC})=0.4680]$. This analysis included the yeast MCM1 protein as the outgroup (Fig. 1A). The tree thus generated was largely similar to one where the Arabidopsis AGAMOUS protein was used as the outgroup (data not shown). Interestingly, while OsMADS1 belongs to the API/AGL9 lineage, it clearly forms a distinct subgroup with the maize genes ZMM8 and ZMM3. Furthermore, OsMADS1 and ZMM8 are the two rice and maize genes that are most closely related in sequence $(80.97 \%$ overall identity and $100 \%$ identity in MADS box). In addition, OsMADS5, a closely related rice gene, also belongs to this sub-group. Notably, this sub-group is distinct from the previously designated orphan group of genes typified by Arabidopsis AGL6 and AGL13 genes. The OsMADS1 sub-group seems to be unique to the monocots with no sequence homologues as yet from any dicot species, despite the near complete sequence information from the Arabidopsis genome. Phylogenetic relationships using distance-based criteria were also determined and this was performed for nucleotide sequences corresponding to the MIK region of the derived proteins. The data was again used to generate a tree with $\mathrm{NJ}$ where bootstrap values were used to indicate the reliability of the branches (see Materials and methods). This analysis deduced a lineage similar to that obtained from analysis of the predicted protein (Fig. 1B). Thus, both analyses place $O s M$ $A D S 1$ and its likely maize homologues in a monophyletic sub-group that has diverged from the AGL9 clade. The topology of the tree derived with the distance matrix correlates well with the cladogram derived using protein sequences for the entire gene.

Different domains of plant MADS-box genes show differing rates of non-synonymous substitutions, with the 
Table 1 Estimates of non-synonymous nucleotide substitution rates within MADS, I and K domains of pairs of genes. OsMADS1 is compared to $A G L 9$, or $A G$-related factors (MIK MADS intermediate keratin-like domain)

\begin{tabular}{llll}
\hline & MADS & K-box & MIK \\
\hline OsMADS5 & 0.020 & 0.095 & 0.091 \\
ZMM8 & 0.000 & 0.036 & 0.050 \\
ZMM3 & 0.015 & 0.079 & 0.077 \\
TDR5 & 0.091 & 0.353 & 0.290 \\
ZAP1 & 0.080 & 0.458 & 0.361 \\
RAP1b & 0.091 & 0.392 & 0.328 \\
AP1 & 0.136 & 0.499 & 0.401 \\
AGL9 & 0.060 & 0.337 & 0.278 \\
AGL13 & 0.079 & 0.510 & 0.370 \\
OsMADS3 & 0.123 & 0.737 & 0.459 \\
\hline
\end{tabular}

lowest degree of change occurring in the MADS box which is constrained by its DNA binding property. Comparing the levels of non-synonymous substitutions can provide clues on functional divergence of specific domains for members of this family. We have compared the proportion of non-synonymous substitution in the gene pairs OsMADS1 versus ZMM8, OsMADS5, ZMM3, AP1 or $A G L 9$. In this analysis we have taken the maize ZAP1, rice $R A P 1 b$ and $O S M A D S 3$, tomato TDR5 and Arabidopsis AGL13 as divergent sequences (Table 1). Despite accounting for the constraints on the MADS box, these analyses suggest $O S M A D S 1$ to be distinct from other molecules of the $A P 1$ or $A G L 9$ clade and its divergence possibly reflects its differing functional specificity. Significantly, all of these analyses place this sub-group as one that is different from that of AGL13 and AGL6. Collectively, these data hint at a unique function for OsMADS1 and $O S M A D S 5$ in rice panicle and flower development.

Expression profile of $O S M A D S 1$ during panicle development

AP1, AGL9 and its likely orthologues from Antirrhinum, Petunia and tomato are the best characterized among the genes in this group. APl is a floral meristem identity gene and the earliest amongst the MADS-box genes to be expressed exclusively in floral tissues. Its temporally early expression in very young floral primordia is uniform and precedes the onset of expression of the other floral organ identity genes. The temporally later expression of $A P 1$ is only in the perianth structures: whorls 1 and 2 (Mandel et al. 1992b). This spatial restriction occurs concomitant to the expression of the organ identity MADS-box gene $A G$ in presumptive whorls 3 and 4 (Gustafson-Brown et al. 1994). In contrast, AGL9 as well as its putative Petunia and tomato orthologues FBP2 and $T M 5$, are expressed uniformly in the young floral meristem. However, the onset of AGL9 expression is after that of $A P 1$. In more mature flowers undergoing organogenesis the $A G L 9$ group of genes are expressed in three inner whorls: petals, stamens and carpels (Angenent et al. 1992; Pneuli et al.1994; Mandel and Yanofsky 1997).
Other members of this clade are expressed in all four whorls of the flower. Not surprisingly, the rice genes such as OsMADS24 and OsMADS45 that are closely related to $A G L 9$ (Fig. 1A) share similarities in expression profiles with these dicot genes. OsMADS45 and OsMADS24 are expressed in lodicules, stamens and carpel of rice flowers (Greco et al. 1997).

Initial studies on $O S M A D S 1$ expression revealed high levels of expression in the flower primordium, a feature similar to that of $A P 1$ - and AGL9-like factors (Chung et al. 1994; Vijayraghavan 1996). The developmentally later expression pattern of OSMADS1 differs in that it is expressed strongly in lemma, palea and weakly in the carpel. The lemma and palea are modified glumes, suggested by certain criteria to be analogous to sepals of dicot flowers (Schmidt and Ambrose 1998). The rice flower bears additional pair of glumes peripheral to the lemma and palea. We have examined whether OsMADS1 is a likely determinant of lemma/palea cell fate by examining in detail its expression pattern in the developing panicle, particularly in very early flower primordia where the outer glumes are established, but the lemma and palea are yet to be formed. We observe that OsMADS1 RNA is first detected in the incipient floral primordium where it is excluded from outer sterile glumes that subtend the flower primordia (Fig. 2A, B, and C, D). OsMADS1 is not expressed in earlier stages of panicle development, i.e. during primary or secondary rachis branch formation (Fig. 2A, B). Its earliest expression occurs well after differentiation of the panicle branches. Therefore, OsM$A D S 1$ and RAP1A are currently the earliest markers to be expressed exclusively in the rice floral meristem (Fig. 2 and Kyozuka et al. 2000). As reported earlier, the early uniform expression of OsMADS1 in the wild-type floral meristem (Fig. 2C, D) is later confined to strong expression in the lemma and palea primordia at about the stage of their initiation (Fig. 2E, F). OsMADSI RNA is completely excluded from the lodicule and stamen primordia and spikelets where organ differentiation is occurring (Fig. 2E-J). Notably, weak but clearly detectable levels of OSMADS1 RNA are seen in the carpel primordia (Fig. 2E, F, and $\mathrm{G}, \mathrm{H}$ ). In contrast, RAPlA is expressed in the lemma, palea and lodicules, with low levels of RNA in the sterile glumes (Kyozuka et al. 2000). These differences in expression patterns reinforce the differences between these two genes hinted at by our phylogenetic analysis (Fig. 1). Prior suggestions of distinctions between the lemma (bract) and palea (prophyll; reviewed in Schmidt and Ambrose 1998) are not reflected in the expression pattern of OSMADS1 which is indistinguishable in these two structures. In this aspect, OsMADSI and RAP1A are similar. The developmentally late expression pattern of OSMADS1 in the lemma, palea and carpels of rice flowers varies from the known pattern of expression for AGL9 group of MADS-domain proteins, including those known in rice. 

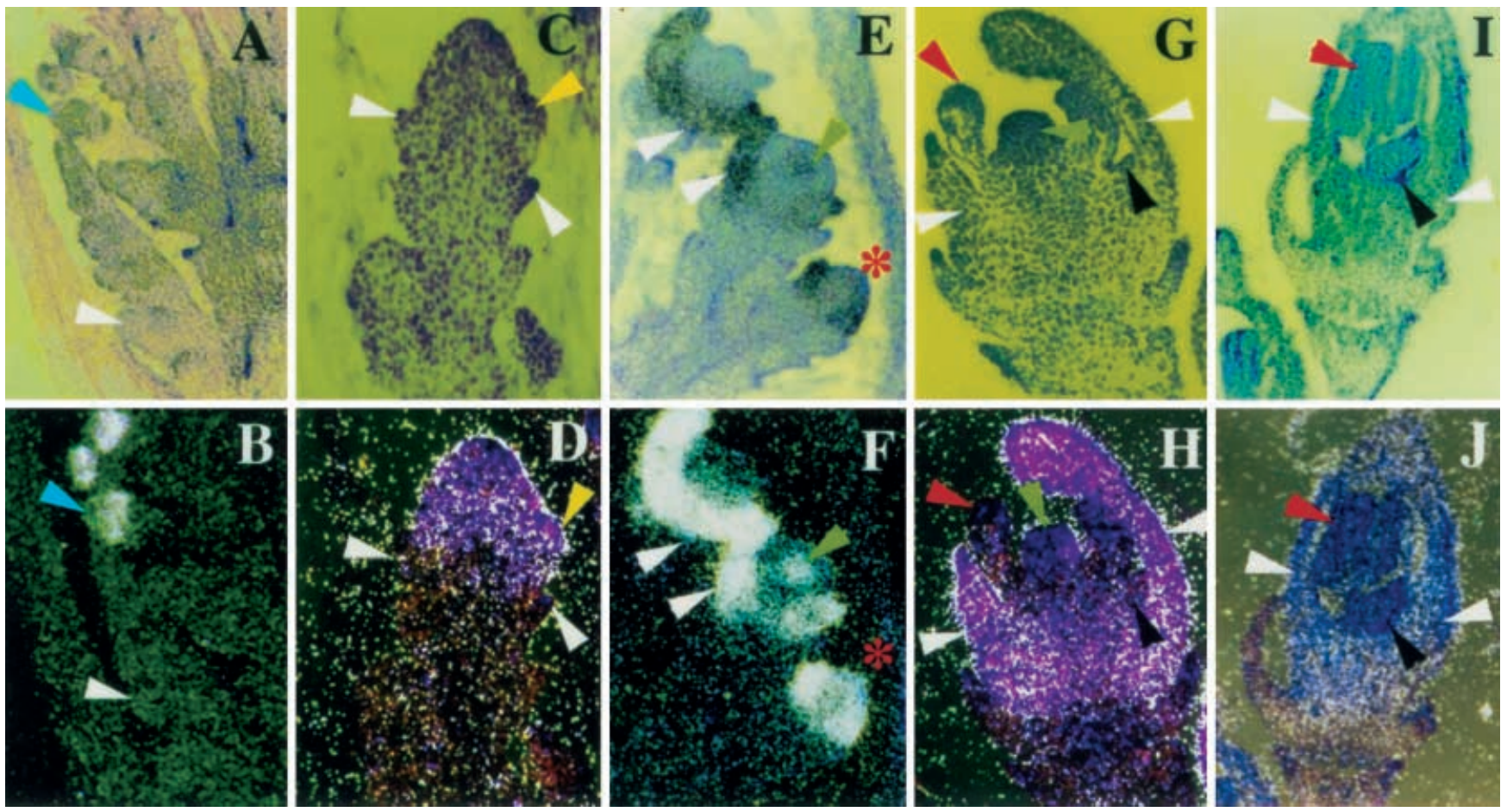

Fig. 2A-J The RNA expression pattern of OsMADS1 in secondary rachis branch primordia $(s r b)$, very young flower primordia, and flower primordia with differentiating organs. In situ hybridization of longitudinal sections of wild-type tissues with an $O s M$ $A D S 1$-specific mRNA probe is shown. A, C, E, G, I Bright-field photographs; the corresponding dark field images are $\mathbf{B}, \mathbf{D}, \mathbf{F}, \mathbf{H}$ and J. RNA expression was detected as the silver grains in the dark field images. A, B Young panicle with developing srb (white arrowhead) and young spikelet primordia (blue arrowhead). C, D Spikelet primordium at the stage of lemma specification; outer rudimentary glumes (white arrowheads) and lemma primordium (yellow arrowhead). E, F Spikelets during organ primordia specification; young spikelet primordium (red star) slightly later in development than that in $\mathbf{C}$, lemma/palea (white arrowhead), and carpel primordium in an older spikelet (green arrowhead). G, H Spikelet with differentiating organ primordia; lemma and palea (white arrowhead), lodicule (black arrowhead), stamen (red arrowhead), and carpel (green arrowhead). I, J Spikelet undergoing anther differentiation; lemma and palea (white arrowhead), lodicule (black arrowhead), and anther lobe (red arrowhead)

Ectopic expression of OsMADS1 affects the panicle morphology

In wild-type plants (TP309), the inflorescence stem develops as a central rachis bearing 6-8 nodes. Each of these nodes produces primary rachis branches (prb) that in turn bear 6-9 nodes. Basal nodes of the prb bear secondary rachis branches (srb), while apical nodes bear individual spikelet primordia. A spikelet primordia in rice bears a single bisexual flower, and these primordia are also generated on the srb (Hoshikawa 1989). A single spikelet in rice contains a pair of sterile glumes that remain small and underdeveloped (Fig. 4A) compared to that observed in other cereal flowers, for example, sorghum, wheat or oat. Internal to these glumes, the rice spikelet bears a single flower with a lemma, a palea, two
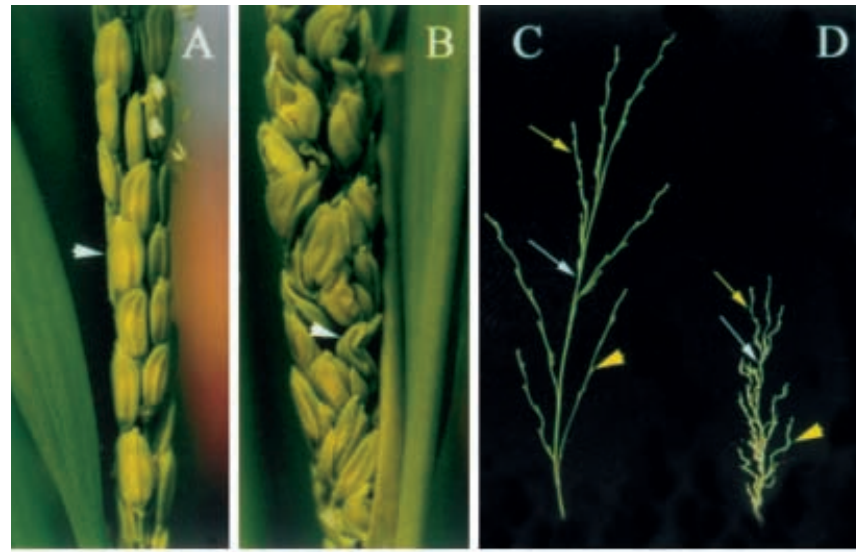

$\mathrm{E}$

OsMADSI

ACT1

Fig. 3 Morphology of panicles from wild-type plants (A) versus pUBI-OsMADS1 transgenic plants (B) at the stage of panicle heading for the wild-type plant. The stunted panicle in the T0 transgenic plant remained embedded in leaf sheath. The disorganized positioning of the spikelets gives a severely twisted phenotype (white arrowhead in B). C A fully emerged wild-type panicle where seeds/spikelets are removed to display the rachis branches. White arrow central rachis, yellow arrow primary rachis branch, yellow arrowhead secondary rachis branch. D T0 OsMADS1 transgenic panicle with spikelets removed to show the severely shortened central rachis and branches thereof. E RT-PCR analysis of OsMADS1 transcripts in transgenic lines. Total RNA from leaves of transgenic plants were used in RTPCR with primers for either the 541-bp C-terminal fragment of $O s M$ ADS1 (upper panel) or the 588-bp fragment of the constitutively expressed endogenous rice ACT1 (lower panel). Lane 1 RT-PCR product from vector pCAMBIA 1381 Xc-transformed plants. Lanes 2-5 RT-PCR products from four independent Ubi-OsMADS1 lines 

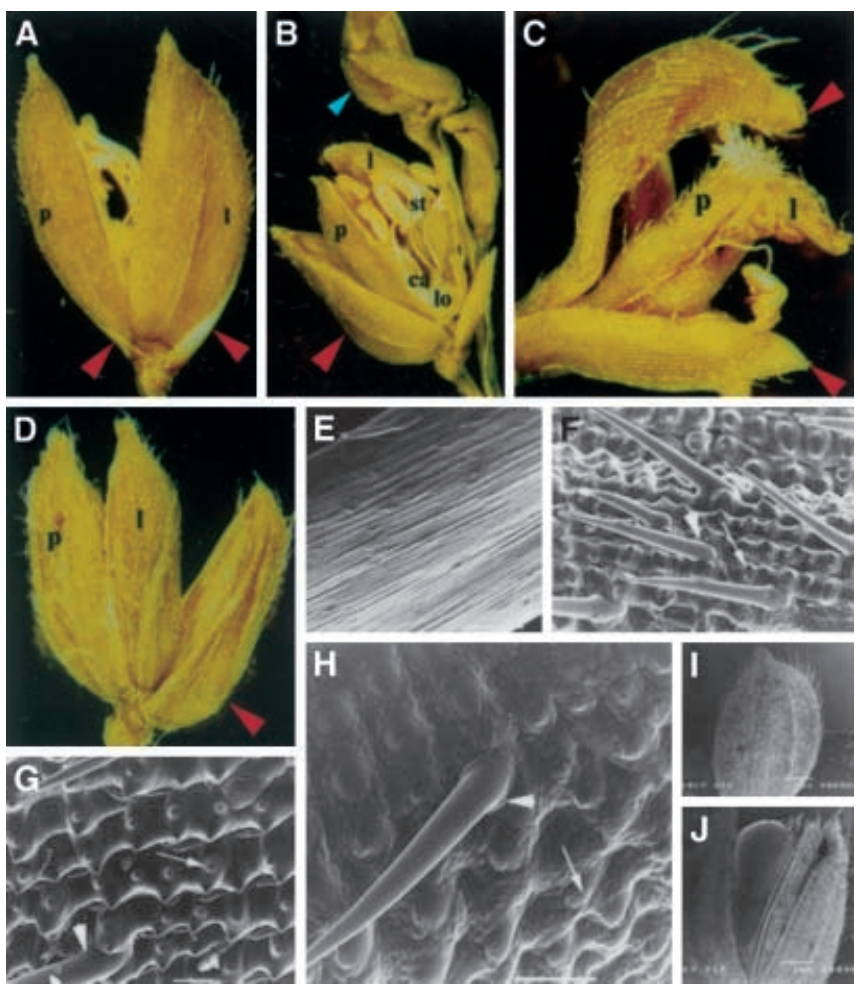

Fig. 4A-J Homeotic phenotypes of OsMADS1 transgenic spikelets. A Wild-type spikelet with rudimentary outer glumes (red arrowheads), lemma $(l)$ and palea $(p)$. B A portion of a T0 transgenic panicle, with one spikelet partially dissected open; the outer glume (red arrowhead) is converted into a lemma/palea-like organ. The other rudimentary glume, while enlarged, is smaller than the first one. Inner to these glumes are the lemma, palea, lodicule (lo), stamens $(s t)$ and carpel $(c a)$. A deformed spikelet with premature termination of differentiation is shown on the same panicle branch (blue arrowhead). C Spikelet where both the glumes (arrowheads) are enlarged and transformed into lemma/palea-like organs. D Spikelet with one outer glume, converted into a lemma/palea-like structure. Internal to this are found the normal lemma and palea, but there are no further internal organs. E-G Scanning electron micrographs (SEM) of the outer surface of rudimentary glume $(\mathbf{E})$, lemma $(\mathbf{F})$, and palea (G) of a wild-type spikelet. H SEM of the epidermal cell surface of a transformed outer glume in a T0 spikelet. White arrows in $\mathbf{F}, \mathbf{G}$, and $\mathbf{H}$ point to several epidermal cells with protrusions typical of lemma/palea and white arrowheads indicate trichomes. I SEM of a wild-type seed enclosed by the lemma and palea. $\mathbf{J}$ Transgenic seed partially enclosed by the lemma/palea and transformed glumes. Scale bars $50 \mu \mathrm{M}$ in $\mathbf{E}-\mathbf{H}$ and $1 \mathrm{~mm}$ in $\mathbf{I}, \mathbf{J}$ lodicules, six stamens and a central carpel. For the sake of convenience, we have used the term rice flower primordium and rice spikelet primordium interchangeably here. We have used ectopic expression studies as a tool to examine the functional contribution of $O s M A D S 1$ to rice panicle and spikelet formation.

An OsMADSI cDNA clone was isolated from the rice panicle cDNA library and then sub-cloned under maize ubiquitin promoter to generate pUbi-OsMADS1 construct (see Materials and methods). This construct was transformed by Agrobacterium-mediated transformation into embryogenic rice calli. Plants from four independent hygromycin-resistant calli were regenerated. During vegetative development, plants transgenic for OsMADS1 did not differ from control vector transformed plants. The parameters of plant height, leaf development and number of tillers per plant (branches from basal vegetative nodes) remained similar to those in controls. Panicle heading was also comparable. A reduction in flowering time by a few days and overall dwarfing upon OSMADSI expression was recently reported by Jeon et al. (2000). The differences between our observations may lie in the photoperiods during transgenic plant growth. This possibility can be tested to examine if OsMADSI contributes to flowering time in a photoperiod-sensitive pathway.

All the plants from four independent Ubi-OsMADS1 lines show defects in panicle development. The panicles are shorter in length, severely defective for internode elongation, and have abnormally positioned branch primordia or lateral primordia on the main axis (Fig. 3C vs D). The panicle length is reduced to about $60 \%$ of that in control plants (Fig. 3C vs D, and Table 2). This is one of the two general features noted in the recent study of Jeon et al. (2000). We detail here our further characterization of the panicle defects. Significant reduction in length of prb, which are in average only $65 \%$ of the length of control branches, was observed (Table 2). Additionally, a striking finding is that the prb are also highly deformed in shape with lateral primordia (i.e. srb primordia and flower primordia) arising at irregular and atypical intervals (Fig. 3D). While the panicle rachis branches are atypical in these plants the number of nodes remains the same as in control panicles (Table 2). The disorganized

Table 2 Panicle characteristics in control vector-transformed plants $(1381 X c)$ or pUbi-OsMADS1-transformed plants (prb primary rachis branches)

\begin{tabular}{|c|c|c|c|c|c|}
\hline Transgene & $\begin{array}{l}\text { Length of } \\
\text { panicle in } \\
\mathrm{cm} \pm \mathrm{SD} \text { a }\end{array}$ & $\begin{array}{l}\text { Number of nodes } \\
\text { on central } \\
\text { rachis } \pm \text { SD }\end{array}$ & $\begin{array}{l}\text { Length of prb } \\
\text { in } \mathrm{cm} \pm \mathrm{SD}\end{array}$ & $\begin{array}{l}\text { Number of nodes } \\
\text { on prb } \pm \text { SD }\end{array}$ & $\begin{array}{l}\text { Number of } \\
\text { spikelets on each } \\
\text { panicle } \pm \text { SD }\end{array}$ \\
\hline 1381 Xc no. 1 & $11.5 \pm 1.3$ & $6.0 \pm 0.8$ & $5.6 \pm 0.4$ & $8.0 \pm 0.5$ & $68.0 \pm 5.9$ \\
\hline $1381 \mathrm{Xc}$ no. 2 & $10.9 \pm 1.0$ & $6.7 \pm 0.7$ & $4.7 \pm 0.6$ & $7.2 \pm 0.4$ & $64.0 \pm 6.0$ \\
\hline 1381 Xc no. 3 & $12.1 \pm 1.0$ & $6.2 \pm 0.8$ & $6.0 \pm 0.6$ & $7.6 \pm 0.4$ & $62.0 \pm 5.8$ \\
\hline $1381 \mathrm{Xc}$ no. 4 & $11.3 \pm 0.9$ & $6.4 \pm 0.8$ & $6.3 \pm 1.0$ & $7.0 \pm 0.5$ & $71.0 \pm 5.6$ \\
\hline pUbi-OsMADS1 no. 1 & $5.9 \pm 0.9$ & $6.0 \pm 0.7$ & $2.1 \pm 0.5$ & $6.0 \pm 0.7$ & $61.2 \pm 5.2$ \\
\hline pUbi-OsMADS1 no. 2 & $4.7 \pm 1.0$ & $5.6 \pm 0.6$ & $2.7 \pm 0.5$ & $7.2 \pm 0.8$ & $59.1 \pm 6.0$ \\
\hline pUbi-OsMADS1 no. 3 & $5.2 \pm 0.9$ & $6.6 \pm 0.7$ & $2.4 \pm 0.4$ & $5.9 \pm 0.6$ & $64.2 \pm 5.8$ \\
\hline pUbi-OsMADS1 no. 4 & $4.9 \pm 0.8$ & $5.9 \pm 0.8$ & $2.6 \pm 0.4$ & $6.7 \pm 0.8$ & $66.2 \pm 5.1$ \\
\hline
\end{tabular}

a Panicle length is represented as length of central rachis. At least seven panicles of each line were examined 
arrangement of the flowers on the shortened panicle and rachis branches contributes to its compact appearance (Fig. 3B). Spikelet morphology was also affected; these data are discussed in the following section. To establish that the panicle phenotype results from ectopic expression of OSMADS1 and not co-suppression (silencing) of the endogenous OsMADSI gene, we have determined the steady state levels of OSMADS1 RNA in vegetative tissues of these transgenic lines. The ubiquitin promoter is expected to direct constitutive expression of OsMADS1 RNA in these transgenic lines. RT-PCR was carried out to measure OsMADS1 RNA levels as compared to those of the endogenous rice actin mRNA. We found a high level and stable expression of OSMADSI RNA that was quantitatively comparable to that of actin $(A C T 1)$ mRNA (Fig. 3E). Together, these results suggest that ectopic expression of OsMADS1 is responsible for the stunted transgenic panicles with their distinct phenotypes. Recently, it has been suggested that floral homeotic genes differentially regulate the cell division patterns during various stages of Arabidopsis flower development (Jenik and Irish 2000). Possibly, the ectopically expressed OsMADS1 exerts its effects on the early stage of panicle branching by altering patterns of cell proliferation.

OsMADS1 ectopic expression results in homeotic transformation of outer glumes into lemma/palea-like organ

As mentioned earlier, rice spikelet has a pair of rudimentary outer glumes at its base, inner to which are the larger, well developed modified glumes: the lemma and palea. These latter organs enclose the internal three whorls of floral organs. By the criteria of OSMADS1 gene expression pattern, and also that of rice RAPIA or the maize ZAPl, it is inferred that lemma and palea define floral organs analogous to first whorl sepals of other flowers (Chung et al. 1994; Mena et al. 1995; Vijayraghavan 1996; Ambrose et al. 2000; Kyozuka et al. 2000). A different line of support for this hypothesis comes from phenotypic analysis of a maize mutant: Silkyl, where lodicules are replaced by lemma/ palea structures; a transformation consistent with a class B loss-of-function mutation (Ambrose et al. 2000). The reasonable prediction is that in grass flowers orthologues of the dicot ABC floral organ identity genes pattern the lemma/palea, lodicules, stamens and carpels. Since the OSMADS1 gene, by sequence relatedness and by expression pattern differs from the abovementioned molecules, we anticipated that the consequences of its ectopic expression on spikelet structure would be informative vis-a-vis its function.

A consistent phenotype in a significant proportion $(\sim 42 \%)$ of flowers in all independently generated OsMADS1 transgenic lines was a drastic enlargement of one pair of the outer glumes. The normally rudimentary glumes immediately peripheral to the lemma and palea now approach the size of the lemma or the palea (Fig. 4C). The normal lemma and palea are found inner to these transformed glumes. A weaker phenotype observed was flowers wherein only one glume was transformed to a lemma/palea like structure; the other glume remained at its normal size. Internal to the transformed glume was the lemma and palea, yet many of such flowers did not bear any stamen or carpel (Fig. 4D). However, a small proportion of the flowers displayed transformation of one glume to a lemma/palea like structure, with otherwise normal floral organ patterning (Fig. 4B). Thus, the most notable phenotype was the presence of over-developed outer glumes, a characteristic also seen by Jeon et al. (2000). We have examined further if these over-developed glumes are merely the result of increase in glume size, or whether they originate from homeotic transformation of a pair of normally rudimentary glumes to lemma/palea. We have determined the epidermal cell surface characteristics of the rudimentary glume, lemma and palea in wild-type flowers and then used their distinguishing features to determine the organ and cellular identities in the over-developed glumes of transgenic flowers. The rudimentary glume bears epidermal cells organized as long files of smooth cells with few trichomes present only on the edges (Fig. 4E). The lemma is typified by epidermal cells with rounded projections and also by a high density of trichomes (Fig. 4F). The wildtype palea bears similar cell morphology as the lemma but has fewer trichomes (Fig. 4G). The epidermal cells of the transformed glume of transgenic flowers has characteristics of both lemma and palea, i.e. cells with rounded epidermal projections and interspersed trichomes (Fig. 4H). These features prove the homeotic transformation of glumes to lemma/palea. Thus OsMADS1 expression in rudimentary outer glumes is sufficient for their conversion into lemma-palea like organs. Possibly, $O s M$ $A D S 1$ achieves this function by targeting downstream genes needed for differentiation of lemma and palea cell types. A speculative function for the Arabidopsis AGL9 like genes is that they act as cofactors for $\mathrm{B}$ and $\mathrm{C}$ floral organ identity genes (Pelaz et al. 2000). The closely related rice genes OsMADS24 and OsMADS45 (Fig. 1) could function similarly and contribute to lodicule, stamen and carpel differentiation in rice. In a similar manner, a plausible hypothesis for OsMADS1 action in the sterile glumes is as a partner/cofactor for gene/s expressed in these glumes. RAPlA, which is expressed at low levels in outer sterile glumes, could be one such candidate partner. In this scenario, ectopic expression of OsMADS1 possibly facilitates the transformation of glumes to lemma/palea like organs. However, we do not observe any transformation of the lodicule in transgenic Ubi-OsMADS1 plants. Thus, it is probable that in lodicules, interaction of RAPIA with other homeotic genes precludes the effects of OSMADS1 ectopic expression therein. Studying the consequences of loss-of-function and gain-of-function of RAPIA in Ubi-OSMADS1 transgenic lines can test these hypotheses.

Since the total number of the flowers in these panicles is similar to that in control panicles (Table 2) we conclude that the flowers which do not bear any internal or- 
gans are not supernumerary flowers generated as a consequence of ectopic expression of OsMADSI. Instead, the data suggest that ectopic expression promotes the precocious assignment of floral meristems on the branches, perhaps at the expense of branch primordia differentiation. Additionally, because there is a sizeable fraction of flowers with underdeveloped floral organs, particularly those with no carpels, we hypothesize that increased expression of OSMADS1 (gain-of-function) promotes increased determinacy of fourth whorl cells. This speculation is supported by observations on phenotypic consequence of OsMADS1 loss-of-function. The lhs loss-offunction mutant arises from mutations in the MADS box of OSMADS1, and the consequences are under-developed leafy lemma and palea, leafy lodicules, decreased stamens and occasional additional carpel or flower (Jeon et al. 2000). However these mutant flowers have normal rudimentary outer glumes as in wild type. Unlike lhs lossof-function mutant, Ubi-OSMADS1 spikelets show homeotic conversion of outer sterile glumes into lemma -palea like organs without affecting the lodicule morphology. These contrasting phenotypes are consistent with a role for OsMADSI and possibly a partner gene expressed in outer glumes in assigning lemma/palea fate. In strong loss-of-function $t h s$ alleles, internal flowers are generated within a spikelet. These observations suggest a loss-of determinacy in the flower primordium in these mutants. These data, together with our observations of underdeveloped carpels in pUbi-OsMADS1 spikelets, implies that OsMADS1 plays an important role in conferring determinacy to the floral meristem center i.e., the fourth whorl. They also suggest that OSMADS1 possibly functions as a floral meristem as well as organ identity gene, though the mechanism by which it defines the floral meristem remains to be examined.

The closely related OsMADS5 gene, whose product shares $72 \%$ identity with OSMADS1, perhaps shares some functions with OsMADS1 (Kang and An 1997). Preliminary studies on the consequences of its ectopic expression in a heterologous system: tobacco, showed weak early flowering (Kang and An 1997). No data was gathered on floral organ types. The individual and combined contributions of OsMADS1 and OsMADS5 can be examined in rice plants that bear loss-of-function/gain-of-function alleles for both loci. The genes ZMM8/ZMM3 could be the orthologues in maize for the OsMADS1/OsMADS5 genes, and may similarly be involved in functions distinct from that of previously described AP1 or AGL9 family of genes. Since no Arabidopsis gene that is closely related to this sub-group has been found as yet, the role of such factors in dicot flowers remains to be investigated.

Acknowledgements We thank Prof. N.V. Joshi, Center for Ecological Sciences, IISc. for his critical thoughts and comments on the phylogenetic analysis of OsMADS1. We thank the Bioinformatics Center, IISc. for the computing facilities. U.V. acknowledges the grants from the Department of Science and Technology, Government of India and The Rockefeller Foundation, USA, during the course of the study.

\section{References}

Alvarez-Buylla ER, Pelaz S, Liljegren SJ, Gold SE, Burgeff C, Ditta GS, Ribas de Pouplana L, Martinez-Castilla L, Yanofsky MF (2000) An ancestral MADS-box gene duplication occurred before the divergence of plants and animals. Proc Natl Acad Sci 97:5328-5333

Ambrose BA, Lerner DR, Ciceri P, Padilla CM, Yanofsky MF, Schmidt RJ (2000) Molecular and genetic analyses of the Silkyl gene reveal conservation in floral organ specification between eudicots and monocots. Mol Cell 5:569-579

Angenent GC, Busscher M, Franken J, Mol JNM, Tunen AJ van (1992) Differential expression of two MADS box genes in wildtype and mutant petunia flowers. Plant Cell 4:983-993

Bowman JL, Alvarez J, Weigel D, Meyerowitz EM, Smyth DR (1993) Control of flower development in Arabidopsis thaliana by APETALA1 and interacting genes. Development 119:721743

Christensen AH, Sharrock RA, Quail PH (1992) Maize polyubiquitin genes: structure, thermal perturbation of expression, transcript splicing and promoter activity following transfer to protoplasts by electroporation. Plant Mol Biol 18:675-689

Chung Y-Y, Kim S-R, Finkel D, Yanofsky MF, An G (1994) Early flowering and reduced apical dominance result from ectopic expression of a rice MADS box gene. Plant Mol Biol 26:657665

Chung Y-Y, Kim S-R, Kang H-G, Noh Y-S, Park MC, Finkel DF, An G (1995) Characterization of two rice MADS box genes homologous to GLOBOSA. Plant Sci 109:45-56

Coen E, Meyerowitz EM (1991) The war of the whorls: genetic interactions controlling flower development. Nature 353:3137

Doyle JA (1973) Fossil evidence on early evolution of monocotyledons. Q Rev Biol 48:339-413

Drews GN, Bowman JL, Meyerowitz EM (1991) Negative regulation of the Arabidopsis homeotic gene AGAMOUS by the APETALA2 product. Cell 65:991-1002

Goto K, Meyerowitz EM (1994) Function and regulation of the Arabidopsis floral homeotic gene PISTILLATA. Genes Dev 8:1548-1560

Greco R, Stagi L, Colombo L, Angenent GC, Sari-Gorla M, Pe ME (1997) MADS box genes expressed in developing inflorescence of rice and sorghum. Mol Gen Genet 253:615623

Gustafson-Brown C, Savidge B, Yanofsky MF (1994) Regulation of Arabidopsis floral homeotic gene APETALA1. Cell 76: 131-143

Hiei Y, Ohta S, Komari T, Takashi K (1994) Efficient transformation of rice (Oryza sativa L) mediated by Agrobacterium and sequence analysis of the boundaries of the T-DNA. Plant J 6: $271-282$

Hoshikawa K (1989) Growing the rice plant: an anatomical monograph. Nosan Gyosan Bunka Kyokai (Nobunkyo), Minato-ku, Tokyo, pp 209-235

Irish VF (1999) Patterning the flower. Dev Biol 209:211-220

Jack T, Brockman LL, Meyerowitz EM (1992) The homeotic gene APETALA3 of Arabidopsis thaliana encodes a MADS box and is expressed in petals and stamens. Cell 68:683-651

Jack T, Fox GL, Meyerowitz EM (1994) Arabidopsis homeotic gene APETALA3 ectopic expression: transcription and posttranscriptional regulation determine floral organ identity. Cell 76:703-716

Jenik PD, Irish VF (2000) Regulation of cell proliferation patterns by homeotic genes during Arabidopsis floral development. Development 127:1267-1276

Jeon JS, Jang S, Lee S, Nam J, Kin C, Lee S-H, Chung Y-Y, Kim S-R, Lee YH, Cho YG, An G (2000) leafy hull sterilel is a homeotic mutation in a rice MADS box gene affecting rice flower development. Plant Cell 12:871-889

Kang H-G, An G (1997) Isolation and characterization of rice MADS box genes belonging to the AGL2 gene family. Mol Cells 7:45-51 
Kang H-G, Noh YS, Chung YY, Costa MA, An K, An G (1995) Phenotypic alterations of petal and sepal by ectopic expression of a rice MADS box gene in tobacco. Plant Mol Biol 29:1-10

Kang H-G, Jeon JS, Lee S, An G (1998) Identification of class B and class $\mathrm{C}$ floral organ identity genes from rice plants. Plant Mol Biol 38:1021-1029

Kushalappa MK (1999) Molecular genetic analysis of flower development in rice. Ph.D. thesis, IISc. Bangalore, India

Kyozuka J, Kobayashi T, Morita M, Shimamoto K (2000) Spatially and temporally regulated expression of rice MADS box genes with similarity to Arabidopsis class A,B, and C genes. Plant Cell Physiol 41:710-718

Li WH (1993) Unbiased estimation of the rates of synonymous and nonsynonymous substitutions. J Mol Evol 36:96-99

Li WH, Wu CI, Luo CL (1985) A new method for estimating synonymous and nonsynonymous rates of nucleotide substitutions considering the relative likelihood of nucleotide and codon changes. Mol Biol Evol 23:150-174

Ma H (1994) The unfolding drama of flower development: recent results from genetic and molecular analyses. Genes Dev $8: 745-756$

Mandel MA, Yanofsky MF (1997) The Arabidopsis AGL9 MADS box gene is expressed in young flower primodia. Sex Plant Reprod 11:22-28

Mandel MA, Bowman JL, Kempin SA, Ma H, Meyerowitz EM, Yanofsky MF (1992a) Manipulation of flower structure in transgenic tobacco. Cell 71:133-143

Mandel MA, Gustafson-Brown C, Savidge B, Yanofsky MF (1992b) Molecular characterization of the Arabidopsis floral homeotic gene APETALA1. Nature 360:273-277

Mena M, Mandel MA, Lerner DR, Yanofsky MF, Schmidt RJ (1995) A characterization of MADS-box gene family in maize. Plant J 8:845-854

Mizukami Y, Ma H (1992) Ectopic expression of the floral homeotic gene AGAMOUS in transgenic Arabidopsis plants alters floral organ identity. Cell 71:119-131

Nandi AK, Kushalappa K, Prasad K, Vijayraghavan U (2000) A conserved function for Arabidopsis SUPERMAN in regulating floral-whorl cell proliferation in rice, a monocotyledonous plant. Curr Biol 10:215-218
Parcy F, Nilsson O, Busch MA, Lee I, Weigel D (1998) A genetic framework for floral patterning. Nature 395:561-566

Pelaz S, Ditta GS, Baumann E, Wisman E, Yanofsky MF (2000) B and $\mathrm{C}$ function floral organ identity functions require $S E P A L L$ ATA MADS box genes. Nature 405:200-203

Pnueli L, Harevan D, Broday L, Hurwitz C, Lifschitz E (1994) The TM5 MADS box gene mediates organ differentiation in the three inner whorls of tomato flowers. Plant Cell 6:175186

Purugganan MD, Rounsley SD, Schmidt RJ, Yanofsky MF (1995) Molecular evolution of flower development: diversification of the plant MADS-box regulatory gene family. Genetics 140: 345-356

Reichmann JL, Meyerowitz EM (1997) MADS domain proteins in plant development. Biol Chem 378:1079-1101

Schmidt R, Ambrose BA (1998) The blooming of grass flower development. Curr Opin Plant Biol 1:60-67

Tajima F, Nei M (1984) Estimation of evolutionary distance between nucleotide sequences. Mol Biol Evol 1:269-285

Theissen G, Kim JT, Saedler H (1996) Classification and phylogeny of the MADS-box multigene family suggest defined roles of MADS-box gene subfamilies in the morphological evolution of eukaryotes. J Mol Evol 43:484-516

Vijayraghavan U (1996) Genetic control of flower development. J Biosci 21:379-395

Wagner D, Sablowski RWM, Meyerowtiz EM (1999) Transcriptional activation of APETALA1 by LEAFY. Science 285:582587

Wang ZY, Tobin EM (1998) Constitutive expression of circadian clock associated 1 (CCA1) gene disrupts circadian rhythm and suppress its own expression. Cell 93:1207-1217

Weigel D (1995) The genetics of flower development: from floral induction to ovule morphogenesis. Annu Rev Genet 29:19-39

Weigel D, Alvarez J, Smyth DR, Yanofsky MF, Meyerowitz EM (1992) $L E A F Y$ controls floral meristem identity in Arabidopsis. Cell 69:843-859

Yanofsky MF, Ma H, Bowman JL, Drews G, Feldmann KA, Meyerowitz EM (1990) The protein encoded by the Arabidopsis homeotic gene AGAMOUS resembles transcription factor. Nature 346:35-39 